International Journal of Natural Sciences Research

2021 Vol. 9, No. 1, pp. 17-25.

$\operatorname{ISSN}(e): 2311-4746$

$\operatorname{ISSN}(p): 2311-7435$

DOI: $10.18488 /$ journal.63.2021.91.17.25

(C) 2021 Conscientia Beam. All Rights Reserved.

check for

check for
updates

\title{
INTELLIGENT SYSTEMS FOR THE INTERNET OF THINGS: ESSENCE, PERSPECTIVES AND PROBLEMS
}

\author{
Shafagat \\ Mahmudova \\ SE Department, Institute of Information Technology of ANAS, Baku, \\ Azerbaijan. \\ Email:shafagat_57@mail.ru Tel:+994509777411
}

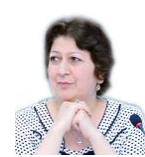

\section{Article History}

Received: 2 September 2021 Revised: 30 September 202 Accepted: 15 October 2021 Published: 4 November 2021

\section{Keywords}

Internet of things

Industrial revolution

Network

Smart devices

Platform.

\section{ABSTRACT}

This article outlines the Internet of Things (IoT). The Internet of Things describes a network of physical objects, i.e., the "thing" including sensors, software, and other technologies for connection and data sharing with other devices and systems over the Internet. In other words, IoT is a relatively new technology enabling many "smart" devices to get connected, to analyze, process, and transfer data to each other and connect to a network. The article clarifies the essence of intelligent systems for the Internet of Things, and analyzes the most popular software for the IoT platform. It studies high-level systems for IoT and analyzes available literature in this field. It highlights most advanced IoT software of 2021 . The article also identifies the prospects and challenges of intelligent systems for the Internet of Things. The creation of new intelligent systems for IoT and the development of technology will greatly contribute to the development of economy.

Contribution/Originality: This analyzes the most popular software for the IoT platform.

\section{INTRODUCTION}

The fourth industrial revolution is often described through basic technologies. These may include a set of future technologies [1, 2]. One of them is the Internet of Things (IoT) [1-3].

The Internet of Things (IoT) describes a network of physical objects, i.e., the "thing" including sensors, software, and other technologies for data connection and sharing with other devices and systems over the Internet.

In other words, IoT is a relatively new technology enabling many "smart" devices to get connected, to analyze, process, and transfer data to each other and connect to a network.

The term IoT was first used in 1999 by Kevin Ashton, a British engineer, founder of the Auto-ID research group $[2,4]$.

IoT connects more and more devices and supports a variety of evolving applications. It is essentially a network where people and devices can communicate with each other, respond to surrounding changes, and make decisions without human intervention. People can configure them or provide access to data, and many people associate IoT with a "smart" house. Technologies and devices developed by Google, Yandex, Amazon, Apple and other companies enable users to shop online, adjust room temperature, turn on lights and music, and give voice commands to virtual assistants. Companies can automate processes and reduce labor costs through IoT. This reduces the amount of unnecessary product waste, improves the quality of services provided, and reduces production and logistics costs. 
Gartner (a research and consulting company specializing in information technology markets) predicts 25 billion devices to be connected to the Internet by 2021. Cybercriminals will continue to attack them, as the IoT system is both a reliable and fast way to spread malware [5].

\section{THE ESSENCE OF INTELLIGENT SYSTEMS FOR THE INTERNET OF THINGS}

The IoT platform is software designed to connect sensors, controllers and other devices and access them remotely.

The most popular software for the IoT platform includes:

- $\quad$ Microsoft Azure IoT.

- Amazon Web Services (AWS) IoT.

- Google Cloud.

- ThingWorx IoT.

- IBM Watson.

- $\quad$ Artik by Samsung Electronics.

- $\quad$ Cisco IoT Cloud Connect.

- $\quad$ Salesforce IoT Cloud.

- $\quad$ And so forth.

Azure IoT involves platform services managed outside and inside the cloud that connect, track and manage billions of IoT assets. It covers data and analysts that will help enterprises develop, deploy, and manage security and operating systems for devices and apparatus, as well as IoT applications [6].

Amazon Web Services (AWS) IoT offers a variety of IoT services, from peripheral devices to the cloud. AWS IoT is the only cloud service designed to combat "polluted" IoT data, connecting appropriate analysts in easy-to-use data management [7].

Google Cloud platform provided by Google is used by consumers working on this infrastructure and designed for their products, such as Google Search and YouTube.

Programming language: Python, Java, Go, C++, Ruby [8].

ThingWorx IoT is a technology platform developed for the Industrial Internet of Things (IIoT). It offers tools and technologies enabling businesses to rapidly develop and apply their powerful, extended and real capabilities $[9]$.

IBM Watson is an IoT for business. Designed for corporate applications, it includes the tools and kits to reduce costs and barriers by IBM and achieve maximum results with strict application of artificial intelligence (AI) [10].

Artik by Samsung Electronics is an open platform designed to accelerate and simplify the development of new IoT applications for industries, organizations and individuals. The platform is a solution with integrated modules equipped with security, cloud integration, local intelligence and various partner applications. Samsung ARTIK is designed to accelerate the development of next-generation IoT devices, solutions and services [11].

Cisco IoT Cloud Connect is new mobile cloud-based software. It is used to provide mobile operators with IoT experiences [12].

Salesforce IoT Cloud offers a platform to store and process data. It is designed to store, regulate and respond to incidents in real time [13].

In terms of technological and technical aspects of IoT development, hardware and software platforms for connecting devices clearly differ; and many experts offer the IoT platform.

Some of the high-end systems for IoT are listed below.

1. ATM keeper, a unique system for physical monitoring of atm systems. The system allows protecting the ATM network from theft, robbery and vandalism. The service is ideal for both small regional networks and large federal networks. 
2. KEEM, a cloud system for tracking objects for business. It is a software package designed to monitor various parameters in a building (industrial and residential buildings), as well as outdoors.

3. Intelligent systems are of great importance for the Internet of Things. The Internet of Things functions as an ordinary information system without intelligent systems. Other technologies include data mining, big data analysis, and other analysis methods created through the Internet of Things.

\section{LITERATURE REVIEW}

1. The Internet of Things (IoT) is significantly dependent on wireless communication devices, as it can detect and interact with other nearby wireless devices. Ease of communication, low cost and launching on market in a short time period of software increase the risk of malware interference. Malicious software can be infect by a large number of network devices using the data sharing between devices, which leads to the creation of botnet, i.e., a network of infected devices controlled by a common malware. The botmaster can be used to launch infrastructure and tools that sabotage the entire network attack. Farooq and Zhu [14] defines a connection between D2D (devices in mobile networks) as a direct connection between the base station (BS) or the main network between two mobile users. It offers an analytical model to study the spread of malware on IoT wireless networks.

2. IoT data is processed and stored using a program known as middleware located on the server. IoT devices send such data through an application-level protocol, which may differ from those supported by mid-level software. This document offers an application layer called MiddleBridge which converts Constrained Application Layer Protocol (CoAP) into message queuing, Message Queuing Telemetry Transport Protocol (MQTT), Data Distribution Service (DDS) and Websockets messages in HTTP. MiddleBridge can be hosted on any computer with Java virtual machine, as all servers are embedded in its code, which allows IoT gadgets to easily transfer data to any REST endpoint. With the proposed approach, it can send a smaller message to middleware (MiddleBridge) that reduces the time of devices' transfer and reconfigures it and sends it to middleware. The created graphical user interface allows users to adjust the respond and transmission of messages during operation. The effectiveness of this approach is assessed by the package size and the response time, taking into account the data sent to the Orion context broker. The results show that the packet size sent by an IoT device via MiddleBridge is 17 times smaller than sending an HTTP request directly to the server, significantly reducing transmission time [15].

3. The Internet of Things (IoT) connects more and more devices and supports a growing variety of applications. The heterogeneity of interfiled and cross-platform device sources is one of the main tasks in the implementation of integrated management and data sharing, which leads to the widescale use of IoT. Inspired by a network defined by the software, the concept of assigned device is offered and the definition and working mechanism in terms of cyber physical mapping are clarified. Based on the concept of the device as a program, the architecture of an open IoT system is developed, which distinguishes top-level applications from the main physical devices (Physical-D) using the SDD mechanism. A logical centralized controller is designed to provide flexible Physical D management and a flexible device detection service and device management interfaces for various application requirements. It also describes an application usage scenario in which SDD-based system architecture can implement unique management, sharing, reuse, recombination and device resource modulation in multiple applications [16].

\section{PERSPECTIVES OF INTELLIGENT SYSTEMS FOR THE INTERNET OF THINGS}

Many successful examples of IoT implementations in foreign practice initiated by both the government and business people are available. For example, in countries such as the European Union, South Korea, China and India, government-supported smart urban technologies are used to increase energy consumption and transport management efficiency. In the United Kingdom and the United States, large-scale programs are implemented to introduce smart meters for remote monitoring of household energy consumption. 
IoT enables businesses to gain a competitive advantage by reducing costs and developing new sources of revenue. For example, the US company GE Aviation produces aircraft engines equipped with sensors ensuring remote access to operational data and determining the optimal algorithms appropriate for the aircraft, which reduces maintenance costs by seven times.

Another example is the Rio Tinto mining company, which operates continuously in Australia and uses unmanned mining machines operated at $1,200 \mathrm{~km}$ from operating center. Industrial IoT technologies are at the focus of Industry 4.0: The German Academy of Sciences and Technology estimates this application to increase the productivity of German industrial enterprises by $30 \%$ by 2025. The consumer market is increasingly using "smart" technologies, for example, according to a results-based PwC survey in the United States, one out of four consumers use smart home devices.

The development of IoT is not only an increase in the introduction of "connected" devices, but also the creation of a technological ecosystem, i.e., a platform enabling data collection, transmission, a set of technological solutions, data processing and implementation of "smart" solutions.

The Internet of Things opens up great opportunities for companies: increasing efficiency, reducing costs, reducing risks, increasing asset reliability and revenue, and more. However, the application of IoT technologies is a complex process requiring a strategy, an implementation plan, and a comprehensive assessment of possible risks and benefits. With knowledge and practical experience, it helps to develop an IoT implementation strategy that best suits the goals of them to be number one in the world of "smart things" and technology.

Figure 1 presents some examples of IoT [17].

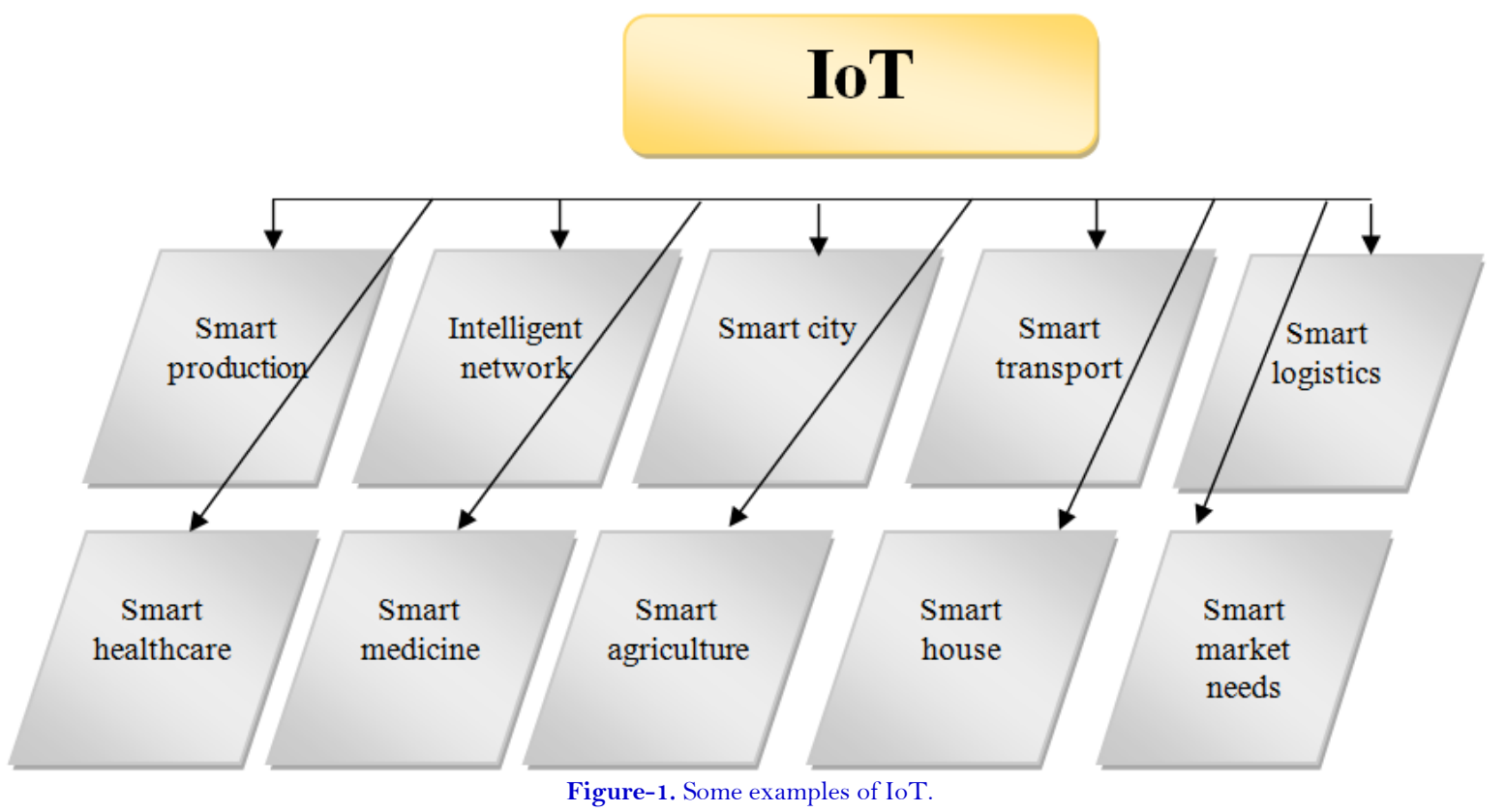

The cost of products of the IoT world is not related to technology or Internet connection. The value is the data collected by smart devices that convert data into action guides for individual owners or groups of people. Therefore, the interface and use of the finished product or software product are of great importance in IoT. All these intelligent things do not belong to only one person; they interact with each other.

Google and Apple have high knowledge and skills in the field of IoT. Both companies are considered to be the most promising areas in the development of IoT. The addition of financial capabilities of "green robots" and the proliferation of iOS and Android devices would make.

Figure 2 Describes the Internet of Things [18]. 


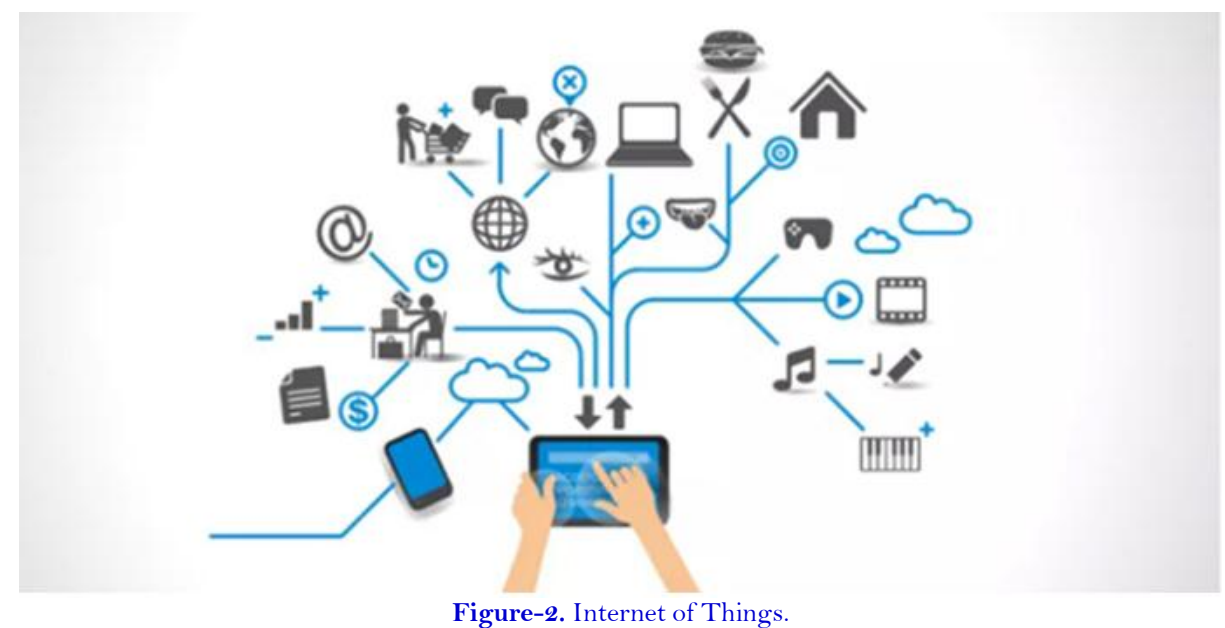

Figure-2. Internet of Things.

To understand how a country is lagging behind, the concept of Industry 4.0 should be reviewed. This includes:

1) Vertical and horizontal digital integration of business processes.

2) Completion of products and services with intelligent modules for their remote interaction.

3) Construction of digital solutions and business models [19, 20].

\section{CHALLENGES OF INTELLIGENT SYSTEMS FOR THE INTERNET OF THINGS}

As in any field, there are many challenges in the field of IoT.

The Internet of Things has opened up a number of possibilities in medicine: when connected to a network, conventional medical devices can sense valuable additional information, provide a broader understanding of symptoms and trends, treat patients remotely, and ensure patients more control over their lives. E-consultancy provides 10 examples of IoT demonstrating the predominant technology in health care [21].

The concept of the Internet of Things is complex, encompassing the interaction of areas such as hardware, networking, and software. Consequently, many problems, both technological and socio-legal challenges arise [22]. One of the main tasks of IoT is to select and develop the right system architecture.

One of the main problems of IoT in modern times is its vulnerability to cyber-attacks.

The risk of unauthorized access to the IoT system is also increasing due to the growing number of interconnected "smart" devices. Many IT companies are now looking for solutions accelerating the full implementation of technology and providing maximum protection against intruders. In 2018, the number of attacks was 12 million. In the first half of 2019 , there were 105 million attacks on IoT devices.

The seven major challenges associated with IoT are listed below [23]:

- Closed Internet.

- Cloud attacks.

- Security problems caused by artificial intelligence.

- Botnet problems.

- Limited AI.

- Lack of reliability.

- Misunderstanding of IoT.

In just a few years, IoT has evolved to an advanced technology of interconnected household things or machines so far. Cisco's review article “The Internet of Everything” estimates the IoT to connect about 50 billion devices by 2020.

If IoT has a problem or weakness, businesses that join it are also at risk. In fact, security is undoubtedly one of the key issues affecting the development; however, there are a number of other issues directly related to it. 
The Internet of Things (IoT) is a new stage in the development of the Internet, significantly expanding the possibilities of data collection, analysis and distribution. In this sense, the Internet of Things is becoming increasingly important.

IoT in ICT.

IoT in industry.

IoT in medicine.

IoT in Telecom.

IoT in household and communal services.

IoT in army.

IoT in Electrical Industry.

IoT in construction.

IoT in logistics.

IoT in agriculture.

And so forth.

An industrial enterprise is a multi-level system and combines sensors and controllers installed at the nodes and departments of an industrial enterprise, tools for transition of collected data and their visualization, and powerful analytical tools for data interpretation [24].

The Internet of Things is a concept in which devices connect to a network and communicate with each other. It helps the devices to be "smart": to independently collect data, share them and make decisions, and so on.

The situation can be monitored and managed through a computer or phone.

The main events of the Internet of Things are as follows:

- Devices accessing the network and communicate with each other.

- Communication method without machines and human intervention.

- Working with large scale data.

- Application of Big Data technologies.

For enterprises, IoT is the basis for collecting data from sources, people and places, analyzing them, and increasing production efficiency [25, 26].

\section{IOT INTELLIGENT SYSTEMS}

IoT must include a large amount of data in the design and construction of intelligent systems, so that it can feel and respond to the user needs.

Software development should take advantage of possible potential, AI \& Machine learning, development and expansion of advanced analytics to convert a data set into templates, predictions, recommendations and actions for exceptionally intelligent interactions and results.

The list of services in this area is listed in Figure 3:

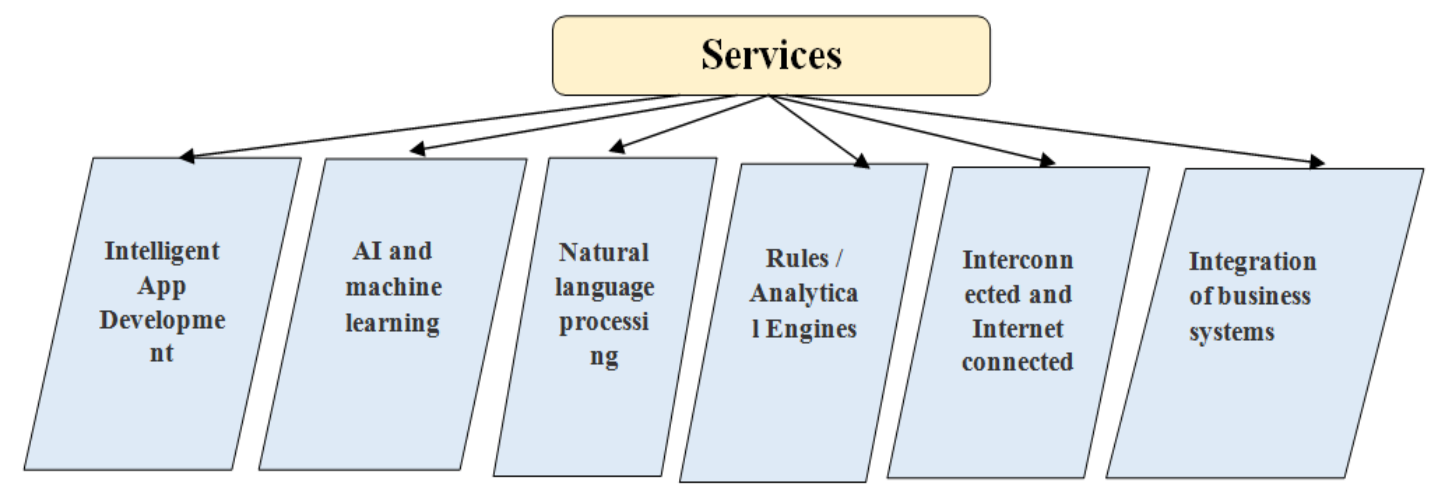

Figure-3. List of services. 
Intelligent App Development; Creates applications with a stable platform of a wide range of technologies, including an analytical engine that manages mono operations with data and working rules, business rules and tracking, control, optimization and IoT support solutions.

AI \&Machine Learning; Creates templates and models using only data and in real time, for example, customer segment, equipment breakdown, etc.

Natural Language Processing; Creates text or voices/bots that can communicate on selected messaging platforms (e.g., Cortana, Alexa, Siri, Google Home) to manage tools. Mobile applications, household appliances, threads, robots and messenger agents.

Rules/Analytical Engines; Develops the kernel of any intelligent SBD system with a flexible, open and powerful analytical engine, and implements the operation rules in sensor and device data to achieve intelligent, manageable and reliable results.

Interconnected \& Internet connected Systems; Connects the devices with other devices or a third-party application. Air, traffic, energy prices, etc. It can be used to add data collected from sensors and IoT devices, which can use external input data sources to create a truly smart and interconnected solution.

\section{ABOUT IOT SOFTWARE}

Below are the top 10 examples of IoT software for 2021.

1. DevicePilot. Pervicepilot device monitoring for close installations provides a glass panel to increase product, operations and customer support groups, increase satisfaction and number, and reduce operating costs.

2. SkySpark. SkyFoundry SkySpark Analytics Platform automatically analyzes data from hardware systems and other smart devices to identify problems, templates, deviations, chances and opportunities to improve operations and reduce costs.

3. Infobip. IoT for global connectivity and control solutions quickly manages systems with automatic order control and alert.

4. Litmus Edge. Litmus Edge is the only flexible and comprehensive IoT platform that provides access to the required data to collect, analyze, manage and integrate data. Litmus collects and normalizes data from all sources.

5. PRTG Network Monitor. PRTG is a solution for comprehensive monitoring, easy-to-use interface and a modern monitoring engine.

6. Blynk. An IoT platform for connecting devices to the cloud, creating applications to manage and control thousands of embedded products.

7. New Relic One.It is the largest comprehensive cloud observation platform.

8. Wolfram Mathematical. It is a technical computing system that provides tools for image processing, geometry, visualization, machine learning, data acquisition and more.

9. Google Cloud IoT. IoT is a current cloud platform that analyzes and connects all devices into a system with a protocol bridge

10. Sematext Cloud. Sematext provides a working view of the subject's records, dimensions, real users, and synthetic data through a complete cloud.

\section{CONCLUSION}

As can be seen from the article, IoT is still evolving. IoT is a concept that makes M2M technology (machineto-machine) more widely used. The M2M segment encompasses the basis of the concept of IoT. In fact, m2m was synonymous with IoT in the early stages of development. At the beginning of 2016, operators also did not have clear criteria for the difference between IoT and M2M.

The creation of new intelligent systems for IoT and the development of technology will greatly contribute to the development of economy. It will lead to the rapid development of smart cities and villages. 
Funding: This study received no specific financial support.

Competing Interests: The author declares that there are no conflicts of interests regarding the publication of this paper.

\section{REFERENCES}

[1] S. Mahmudova, "Study and comparative analysis of programming languages used for big data," Review of Industrial Engineering Letters, vol. 8, pp. 1-9, 2021.Available at: https://doi.org/10.18488/journal.79.2021.81.1.9.

[2] H. Harun and A. Mohd, "A study using internet of things concept toward engineering education," International Journal of Advances in Computer Science and Technology, vol. 4, pp. 133-136, 2015.

[3] M. Sergey, "4-ya promyshlennaya revolyutsiya v Davose. Retrieved from: https://expert.ru/2016/01/21/chetvertayapromyishlennaya-revolyutsiya/. [Accessed 21 yanvar '2016]," 2016.

[4] Z. Al'namer, "Internet of things (IOT): Problems and future directions," Information Technology, vol. 24, pp. 24-26, 2018.

[5] L. Kleymenova, "Chto takoye internet veshchey? RBK Trendy Moscow, Russia. Retrieved from: https://trends.rbc.ru/trends/industry/5db96f769a7947561444f118," 2021.

[6] I. Azure, "Quickly turn your vision into reality with secure, scalable, and open edge-to-cloud solutions from the Microsoft Cloud. Retrieved from: https://azure.microsoft.com/en-us/overview/iot/," 2021.

[7] I. AWS, "Develop intelligent Ut solutions with advanced machine learning and artificial intelligence integrations. Retrieved from: https://aws.amazon.com/ru/iot/," 2021.

[8] Why Google Cloud, "Why Google cloud. Retrieved from: https://ru.wikipedia.org/wiki/Google_Cloud_Platform," 2021 .

[9] Short-Range, "Short-Range wireless solutions to power up industrial IoT. Retrieved from: https://www.ptc.com/en/resources/iiot/product-brief/thingworx-platform," 2021.

[10] IBM Watson Products, "IBM Watson products. Retrieved from: https://www.ibm.com/watson," 2021.

[11] Samsung ARTIK, "Samsung ARTIK. Retrieved https://www.tadviser.ru/index.php/\%Do\%9F\%D1\%80\%Do\%BE\%DO\%B4\%D1\%83\%Do\%BA\%D1\%82:Samsung_AR TIK," 2017.

[12] Suppliers, "Top 40 suppliers. Retrieved from: https://www.iotone.com/suppliers," 2021.

[13] D. Zhinko, "Salesforce, "IoT Cloud: Benefits and limitations. Retrieved from; https:// www.scnsoft.com/blog/salesforce-iot-cloud-benefits-and-limitations," 2021.

[14] M. J. Farooq and Q. Zhu, "Modeling, analysis, and mitigation of dynamic botnet formation in wireless IoT networks," IEEE Transactions on Information Forensics and Security, vol. 14, pp. 2412-2426, 2019.Available at: https://doi.org/10.1109/tifs.2019.2898817.

[15] d. C. MAA, J. J. Rodrigues, P. Lorenz, P. Solic, J. Al-Muhtadi, and V. H. C. Albuquerque, "A proposal for bridging application layer protocols to HTTP on IoT solutions," Future Generation Computer Systems, vol. 97, pp. 145-152, 2019.

[16] P. Hu, H. Ning, L. Chen, and M. Daneshmand, "An open Internet of Things system architecture based on softwaredefined device," IEEE Internet of Things Journal, vol. 6, pp. 2583-2592, 2018.Available at: https://doi.org/10.1109/jiot.2018.2872028.

[17] Vosem' Klyuchevykh, "Vosem' klyuchevykh tekhnologiy Internet veshchey. Retrieved from: https://www.pwc.ru/ru/publications/the-internet-of-things.html," 2021.

[18] Perspektivy Razvitiya, "Prospects for the development of the Internet of Things until 2020. Retrieved from: http://1234g.ru/novosti/internet-veshchej-k-2020-godu," 2019.

[19] R. Perspektivy, "Prospects for the development of the Internet of things. Retrieved from: https://softline.ru/about/news/23584," 2017.

[20] R. Liu and J. Wang, "Internet of things: Application and prospect," presented at the MATEC Web of Conferences 100:02034, 2017. 
[21] A. S. Albahri, J. K. Alwan, Z. K. Taha, S. F. Ismail, R. A. Hamid, A. Zaidan, O. S. Albahri, B. Zaidan, A. Alamoodi, and M. Alsalem, "IoT-based telemedicine for disease prevention and health promotion: State-of-the-Art," Journal of Network and Computer Applications, vol. 173, p. 102873, 2021.Available at: https://doi.org/10.1016/j.jnca.2020.102873.

[22] Problemy i Ugrozy Bezopasnosti IoT, "YoT security problems and threats. Retrieved from: https://center2m.ru/informatsionnaya-bezopasnost-veschey," 2018.

[23] D. Roe, "How edge computing will transform the digital workplace. Retrieved from: https://www.cmswire.com/information-management/how-edge-computing-will-transform-the-digital-workplace/," 2020.

[24] Chto Takoye, "Chto takoye internet veshchey Internet of Things (IoT). Retrieved from: https://www.tadviser.ru/index.php/Интернет_вещей_Internet_of_Things_(IoT)," 2017.

[25] Internet Veshchey, "What is the Internet of things and how can it help businesses earn more ?. Retrieved from: https://habr.com/ru/post/474796/," 2021.

[26] Resheniya Interneta, "IoT solutions for actionable insights. Retrieved from: https://www.hpe.com/ru/ru/solutions/internet-of-things.html," 2020. 